\title{
ARTIGOS
}

\section{Simpósio de Grupos de Pesquisa sobre Formação de Professores do Brasil - Síntese das observações realizadas}

\author{
Maria do Céu Roldão (U.Católica Portuguesa-Porto) \\ Marli Eliza D.A André (PUC-SP) \\ Iria Brzezinski (PUC-GO) \\ Joana Paulin Romanowski (PUC-PR) \\ Emilia de Freitas Lima (UFSCar)
}

\begin{abstract}
RESUMO: Este texto apresenta a síntese do III Simpósio de Grupos de Pesquisa sobre Formação de Professores do Brasil. Foi produzido a partir do registro das observações feitas nos dois dias do evento - 3 e 4 de outubro de 2016 - pelas autoras desta síntese que, como última parte do simpósio, foi socializada e discutida com todos os participantes. A síntese constituiu a base sobre a qual foi organizado o texto, estruturado em cinco seções: composição dos grupos de pesquisa; tendências observadas pela equipe de avaliação nos relatos dos grupos de pesquisa sobre formação de professores; dimensões menos evidentes nesses relatos; financiamentos dos projetos; e, finalmente, referenciais teóricos e conceitos-chave - ampliação e diversificação.
\end{abstract}

PALAVRAS-CHAVE: Formação de Professores. Grupos de Pesquisa. Campo Investigativo

\section{Third Symposium of Research Groups on Teacher Training in Brazil: synthesis of observations conducted}

\begin{abstract}
This text presents the synthesis of the III Symposium of Research Groups on Teacher Training in Brazil. It was produced from the record of the observations made on the two days of the event - October 3 and 4, 2016 - by the authors of this synthesis which, as the last part of the symposium, was socialized and discussed with all participants. The synthesis was the basis on which the text was organized, structured in five sections: composition of research groups; trends observed by the evaluation team in reports from research groups on teacher education; dimensions less evident in these reports; project financing; and, finally, theoretical references and key concepts - expansion and diversification.
\end{abstract}

KEYWORDS: Teacher Training. Research Groups. Investigative Field 


\section{INTRODUÇÃO}

O GT 08 - Formação de Professores, da Associação Nacional de Pós-Graduação e Pesquisa em Educação, realiza simpósios de grupos de pesquisa sobre formação de professores. O I Simpósio foi realizado na Pontifícia Universidade Católica de São Paulo (PUC/SP), de 24 a 25 de julho de 2006, sob a coordenação de Emília Freitas de Lima e Laurizete Ferragut Passos, com a finalidade de promover a discussão sobre a delimitação do campo de formação de professores com base no mapeamento dos grupos de pesquisa que focalizam essa temática no Brasil. Contou com a participação de 73 grupos de pesquisa das diferentes regiões brasileiras.

O II Simpósio teve como local a Pontifícia Universidade Católica do Paraná (PUC/PR), em Curitiba/PR, foi realizado de 24 a 25 de março de 2011, sob a coordenação de Joana Paulin Romanowski e Leny Rodrigues Martins Teixeira. Assumiu como objetivos: (i) analisar junto aos grupos de pesquisa o movimento em que se situa a investigação sobre a formação de professores, visando à compreensão do campo e as tendências investigativas; (ii) favorecer a interação entre os grupos colaborando para a constituição de redes de pesquisa no campo contribuindo para o debate consubstanciado sobre a pesquisa em formação de professores na constituição da área e (iii) Examinar a contribuição social da pesquisa sobre formação de professores para a produção de conhecimento na área da educação e para o sistema educacional brasileiro. Este simpósio contou com a participação de 34 grupos de pesquisa e 224 componentes destes grupos.

O III Simpósio foi realizado de 3 a 4 de outubro de 2016, na cidade de Guarulhos SP, tendo como local o Teatro Adamastor, cedido pela Secretaria Municipal de Educação daquele município. Teve a coordenação de Magali Silvestre e Márcia Hobold, e buscou oferecer um espaço para os pesquisadores apresentarem as pesquisas desenvolvidas pelos seus grupos, ampliando o debate e socializando a produção de conhecimento do campo da educação, mais especificamente no que se refere à formação de professores. Participaram deste simpósio 47 grupos inscritos dos quais 42 compareceram.

A metodologia de realização dos simpósios tem consistido de duas fases. Previamente à sua realização é feita a inscrição dos grupos de pesquisa, da qual constam: (i) a indicação dos participantes do grupo e da instituição à qual ele se vincula; (ii) o envio de um texto escrito, a título de documento-base, contendo: o objetivo principal da pesquisa realizada pelo grupo, o campo temático (tema e subtemas), a população-alvo, o quadro teórico, a natureza da investigação, as contribuições para o campo da formação de professores e as perspectivas teórico-metodológicas.

Na realização do simpósio, num primeiro momento os textos produzidos pelos grupos de pesquisa são socializados. Nesse momento, devido ao elevado número de grupos participantes, é necessária a organização de subgrupos que trabalham em sessões concomitantes, cada uma delas contando com o acompanhamento de membro do GT 08 - Formação de Professores e membros de um grupo de observação/ avaliação. O grupo de avaliadores busca examinar as pesquisas socializadas, de modo a que se chegue a um panorama da situação dos grupos, quanto às temáticas investigadas, os fundamentos teóricos, as abordagens teórico-metodológicas, os procedimentos de investigação e análise e os principais resultados. A síntese resultante da análise integrativa das pesquisas dos grupos aponta as principais contribuições dos estudos, as tendências mais evidentes e sinaliza pontos críticos que devem ser objeto de maiores discussões pelos grupos. Essas 
apreciações têm possibilitado constante aperfeiçoamento dos estudos da área e têm contribuído para o fortalecimento do campo da formação de professores.

Os textos-síntese de cada um dos simpósios têm sido amplamente divulgados, seja pela sua publicação no sítio eletrônico do GT, seja pela socialização com os grupos participantes, ou, ainda, em eventos, reuniões, palestras ou conferências.

Este texto apresenta a síntese do III Simpósio de Grupos de Pesquisa sobre Formação de Professores do Brasil, elaborada pelas pesquisadoras Maria do Céu Roldão (Universidade Católica Portuguesa), Marli André (Pontifícia Universidade Católica de São Paulo), Iria Brzezinski (Pontifícia Universidade Católica de Goiás e Universidade de Brasília), Emília Freitas de Lima (Universidade Federal de São Carlos) e Joana Paulin Romanowski (Pontifícia Universidade Católica do Paraná) que constituíram equipe de avaliação do III Simpósio. Após assistir à apresentação dos trabalhos dos grupos de pesquisa, essa equipe reuniu-se para organizar as observações e registros dos dois dias do evento - 3 e 4 de outubro de 2016 - e produzir a síntese que, como última parte do simpósio, foi socializada e discutida com todos os participantes.

Tal síntese constituiu a base sobre a qual foi organizado o presente texto, estruturado em cinco seções: composição dos grupos de pesquisa; tendências observadas pela equipe de avaliação nos relatos dos grupos de pesquisa sobre formação de professores; dimensões menos evidentes nesses relatos; financiamentos dos projetos; e, finalmente, referenciais teóricos e conceitos-chave - ampliação e diversificação.

\section{COMPOSIÇÃO DOS GRUPOS DE PESQUISA}

Em relação à composição, expressa-se em vários casos o entendimento de que um grupo de pesquisa é formado pelo orientador e seus orientandos. O próprio Diretório de Grupos de Pesquisas do CNPq estabelece em suas orientações e definições de grupo de pesquisa que esta é uma das possíveis composições. Entretanto, as discussões empreendidas pelas associações e círculos de pesquisadores têm ampliado essa concepção, apontado que a evidência cada vez maior da importância das trocas de conhecimentos e da riqueza que perspectivas variadas podem trazer para um determinado campo de estudos, sinalizam a tendência de que os grupos se constituam como espaços mais coletivos, em que professores de diferentes especialidades, com perspectivas diferenciadas, contribuam para o enriquecimento do grupo, seja em seus aspectos teórico-metodológicos, seja no aprofundamento das análises realizadas. Desse modo, compreende-se que caminhar para a constituição de grupos compostos por pesquisadores experientes, pesquisadores em formação e intercâmbios entre vários projetos de pesquisa poderia ser assumido, para uma possível substituição do modelo "grupo pesquisador e orientandos".

Essa proposição coloca em destaque o conceito de grupo de pesquisa, tanto para a organização dos próximos Simpósios do GT 08, quanto como ponto de reflexão para os pesquisadores sobre a composição de seu grupo de pesquisa: qual modalidade será adotada? A do grupo do orientador com seus orientandos? Ou uma modalidade com pesquisadores experientes e iniciantes, estudantes de graduação e de pós, de um programa ou de diferentes programas? São muitas as alternativas. Essa questão da composição dos grupos de pesquisa é algo que ficou para todos, como uma problemática a ser discutida e pensada para o próximo Simpósio.

A partir dessas considerações em torno dos grupos de pesquisa, dos registros realizados durante as sessões do simpósio, alguns pontos estavam presentes nos relatos apresentados e serão indicados a seguir. 


\section{TENDÊNCIAS}

Esta seção é dedicada às tendências observadas pela equipe de avaliação nos relatos dos grupos de pesquisa sobre formação de professores. São elas: maior articulação universidade-escolas públicas; ênfase na prática e significados atribuídos a ela; estudos de narrativas e metodologias baseadas em narrativas; ligação da pesquisa com a intervenção; natureza e clarificação do conhecimento produzido; reforço de parcerias internacionais.

\subsection{MAIOR ARTICULAÇÃO UNIVERSIDADE-ESCOLAS PÚBLICAS}

Uma tendência que nos pareceu evidente, acentuada por Maria do Céu Roldão, que vem acompanhando nossos simpósios e alguns dos grupos de pesquisa, é a presença de uma preocupação com a articulação da Universidade com a Escola Pública, em especial com a Educação Básica.

Essa afirmação se pauta no fato de terem sido apresentados muitos grupos com investigações buscando um diálogo da pesquisa com as escolas públicas de Educação Básica. Isso foi algo diferente em relação ao nosso II Simpósio, pois despontam com mais frequência grupos que estão fazendo um diálogo com as escolas e com as redes de ensino. Além disso, na composição desses grupos, percebemos uma tendência de constituição de grupos colaborativos, compostos por professores da universidade, professores atuantes nas redes de ensino e nas escolas. Essa aproximação entre universidade e escola com maior incidência nos grupos de pesquisa, promovendo o trabalho colaborativo, expressa a tendência de fortalecer a relação com a prática pedagógica nas pesquisas em formação de professores.

Nessa questão de aproximar mais os trabalhos de pesquisa com a prática, notamos que de um lado há um fator bastante positivo no sentido de que a universidade, finalmente, se preocupa mais com a articulação entre o conhecimento acadêmico e o conhecimento da prática. Assim, o conhecimento produzido em pesquisa, de alguma maneira passa a ter mais repercussões na prática educativa escolar.

\section{2 ÊNFASE NA PRÁTICA E SIGNIFICADOS ATRIBUÍDOS A ELA}

A análise dos trabalhos apresentados revelou certa ênfase na prática, mas em um sentido que nos causou preocupação. Parece, em alguns casos, que são estudos da prática pela prática, sem que essa prática seja devidamente interrogada, discutida, e analisada quanto a sua relação com a formação de professores. Falta problematizar essa prática, falta perguntar: por que se estuda essa prática? E o que se busca quanto à formação de professores a partir dessa prática? Que reflexões são provocadas em torno da prática? Que reflexões são feitas sobre a práxis nos estudos acerca da formação de professores? Em alguns momentos nos pareceu, a todas nós, que esta é uma questão que ainda precisa de muita discussão, muita reflexão para não resvalar em praticismo. Tomar a prática de forma superficial como orientadora da pesquisa traria um retrocesso, enfraquecendo o próprio objeto de estudo da formação de professores. A nossa interrogação é a de que carece direcionar a pesquisa para as questões e objeto da formação. Esse é um dos pontos privilegiados na fala de Maria do Céu Roldão, que aborda a questão do campo da formação de professores. Assim, nessa síntese queremos apenas registrar nossa preocupação e assinalar a necessidade de maior discussão sobre a pesquisa da prática pedagógica e sua relação com o campo da formação de professores.

\subsection{ESTUDOS DE NARRATIVAS E METODOLOGIAS BASEADAS EM NARRATIVAS}

Outra tendência nova que percebemos, e que não estava tão marcada nos simpósios anteriores, é o uso de narrativas nos estudos da formação de professores. Constatamos que apareceram muito mais trabalhos 
com narrativas, seja do ponto de vista metodológico, seja epistemológico, do que nos simpósios anteriores. Destacamos que esta abordagem valoriza o professor como produtor de conhecimento, expressando uma contribuição muito positiva, mas também deixa aberta uma questão: nem sempre ficou explicitada a relação entre o uso da narrativa e a formação de professores e mais problemática ainda é a pouca explicitação da pesquisa que foi realizada com as narrativas. Por exemplo: narrar o processo formativo, onde está a pesquisa? Qual a relação entre a pesquisa sobre formação e as narrativas? Ou pode-se perguntar: o que a pesquisa sobre narrativas pode nos dizer em relação ao nosso objeto que é a formação de professores?

\subsection{LIGAÇÃO DA PESQUISA COM A INTERVENÇÃO - SENTIDOS ATRIBUÍDOS}

Questões semelhantes às da pesquisa com narrativas foram observadas nas pesquisas de intervenção. Neste III Simpósio foram apresentadas em menor número do que no simpósio anterior. No outro, houve mais menções ao uso de pesquisa ação, por exemplo. No entanto, ressaltamos que em alguns grupos do simpósio atual, a pesquisa-intervenção foi mencionada nas apresentações. Em uma delas o foco era uma descrição da intervenção realizada, ficando no ar qual teria sido a pesquisa, que conhecimento teria sido produzido a partir dessa intervenção. Essa é a pergunta a ser feita, também, a respeito das pesquisas com narrativas: qual é a contribuição que as narrativas trazem para o conhecimento? Ou que conhecimento produzem? Qual a contribuição para o campo da formação de professores? Essas são questões a serem feitas, porque afinal estamos tratando neste simpósio de grupos de pesquisa. Desse modo a pesquisa tem que refletir e indicar que conhecimento vem sendo produzido, decorrente dessas formas de pesquisar, seja narrativa, seja pesquisa ação ou intervenção. Em alguns relatos de grupos de pesquisa-intervenção foi constado o desenvolvimento de trabalhos de extensão, caracterizados mais como uma ação de extensão do que realmente uma pesquisa de (ou sobre) intervenção.

\subsection{NATUREZA E CLARIFICAÇÃO DO CONHECIMENTO PRODUZIDO}

Nos pontos 2.2, 2.3, e 2.4 se configura a discussão central do processo de pesquisa. Que se entende como conhecimento produzido, ou o que conta como conhecimento na legitimação social, parafraseando Menga Lüdke. Como se produz e o que se procura alcançar em cada uma destas três tendências? Embora muito valiosas, o eixo epistemológico que vimos procurando consolidar no percurso do GT 08 deve ser sempre uma preocupação. Sublinhamos que a produção de conhecimento em formação de professores - como na educação em geral - muito embora se interligue sempre com uma prática, requer a sua desconstrução teórica e a produção de conhecimento analítico que permita a reconfiguração enriquecida de práticas. No caso das narrativas, o seu uso, que tem um potencial extraordinário, tem que passar por este crivo de visibilizar como essa riqueza analítica se opera e a que saber sistemático conduz. Do mesmo modo, produzir uma boa intervenção não pode ser confundido com produzir conhecimento que a sustente, desconstrua e avalie num processo de continuidade.

\subsection{REFORÇO DE PARCERIAS INTERNACIONAIS}

Outra tendência que percebemos são estudos em parcerias com instituições estrangeiras. Grande parte dos estudos tinha colaboradores ou instituições de ensino superior de outros países, em maior número com grupos de pesquisa portugueses e franceses. Em alguns grupos havia participação de colaboradores, consultores de pesquisa internacionais. Sabemos que um dos critérios de avaliação, adotado pelos órgãos de fomento, expansão e consolidação da pós-graduação, é a internacionalização. Esse é um item muito forte nas nossas avaliações, tanto no Brasil como em outros países. Em Portugal há uma política de fomento nesta 
perspectiva. Desse modo, foi registrado que essa política já está se refletindo nos grupos de pesquisa que foram aqui apresentados, pois muitos deles mencionaram essas colaborações internacionais.

\section{DIMENSÕES MENOS EVIDENTES}

Nesta seção vamos abordar o que deixou de aparecer, ou o que foi menos mencionado nas apresentações dos grupos de pesquisa. Foram aqui consideradas cinco dimensões, que são descritas a seguir.

\subsection{INTEGRAÇÃO DOS OBJETOS PARCIAIS DE ESTUDO AO OLHAR SOBRE O CURRÍCULO GLOBAL DA FORMAÇÃO E SUA LÓGICA INTERNA}

Entre os temas e assuntos abordados houve pouca indicação quanto ao currículo da formação inicial, como um currículo fortalecido em torno da formação como um todo. Alguns relatos abordam parte do currículo, ou seja, algumas disciplinas, o estágio, o Programa Institucional de Bolsa de Iniciação à Docência - PIBID. Porém, é fundamental que esses objetos parciais sejam integrados a olhares sobre o currículo global da formação e sobre sua lógica interna. Nessa perspectiva, faz falta uma maior problematização da formação, da composição curricular, dos processos formativos. Percebemos que ainda estão silenciados os estudos nessa direção.

\subsection{CLARIFICAÇÃO DA TIPOLOGIA “ESTUDO DE CASO”, DEMASIADO VULGARIZADA COM ALGUMA PERDA DE RIGOR METODOLÓGICO}

Outra dimensão pouco abordada foi a das pesquisas em grande escala. Em vez disso, tivemos $90 \%$ de estudos chamados de "estudos de abordagem qualitativa", mas são pesquisas de dimensão micro, ou seja, mais pontuais.

Dentre estas, queremos destacar que nos relatos de uso do estudo de caso, algumas vezes não se pode distinguir a abordagem metodológica devidamente. Ora, o estudo de caso tem características, tem peculiaridades para que seja reconhecido como tal. Parece, no entanto, que nem todos os investigadores têm o entendimento das características próprias de um estudo de caso. Esse tipo de pesquisa exige um estudo aprofundado, em que se tenha algo peculiar, importante para ser destacado como estudo de caso. Assim, não é só porque é um estudo delimitado de pequena escala que se caracteriza e se nomeia como estudo de caso. Ainda temos de problematizar essas questões metodológicas em nossas discussões do campo.

\subsection{ESCASSEZ DE ESTUDOS EXTENSIVOS, DE MAIOR ESCALA, COM QUANTIFICAÇÃO DE TENDÊNCIAS}

A incluir como questão, como apontamos anteriormente, é a escassez de estudos de grande escala, estudos mais abrangentes. Trata-se de estudos que permitem uma visão ampla e extensiva de um objeto de estudo, incluem um grande número de sujeitos, cuidados no tratamento dos dados. Essa não utilização de estudos de grande escala pode estar associada ao receio de ser positivista, de que um estudo abrangente é sempre positivista e, isso é um grande equívoco. Faz falta para nossa área ter estudos de maior amplitude, com dados que nos permitam ter uma compreensão mais alargada e ampliada das questões da formação de professores em nosso país. 


\subsection{ESCASSEZ DE ESTUDOS LONGITUDINAIS}

Outro tipo de estudo não mencionado nos relatos dos grupos é o estudo longitudinal, estudo de acompanhamento, que se faz ao longo do tempo. Esses estudos são extremamente importantes, pois permitem seguir os mesmos sujeitos, os mesmos participantes por um período mais longo de tempo. Sabemos da objeção e dificuldade na realização deste tipo de pesquisa, porque os nossos projetos têm um tempo limitado de duração, os projetos financiados têm no máximo três anos, o que ainda é pouco tempo para estudos longitudinais. Por exemplo, Marli André, no estudo que está desenvolvendo, tinha a pretensão de fazer um estudo longitudinal, e em conversa com Maria do Céu Roldão, que é a consultora, esta lhe disse: "eu penso que não dá para vocês fazerem um estudo longitudinal nesses três anos que vocês têm; vai ser muito pouco tempo". Então, há realmente empecilhos à realização de estudos longitudinais. Talvez tivéssemos de planejar melhor: partir de um estudo para dar continuidade em outro, com os mesmos sujeitos, ou seja, fazer um seguimento, acompanhar ao longo do tempo.

\subsection{AUSÊNCIA DE ESTUDOS SOBRE FORMAÇÃO CONTINUADA}

Entre os temas pouco frequentes situa-se o da formação continuada, os processos de formação continuada, como um assunto do campo da formação de professores. Percebemos que se fala sobre a formação continuada, mas não se pesquisam os processos dessa formação, como uma temática pertinente ao campo. No III Simpósio, sentimos falta de ver contempladas, com mais intensidade, as interrogações sobre a formação continuada. O que esses processos formativos estão nos dizendo? Os programas em nível regional, estadual, nacional, e assim por diante.

\section{FINANCIAMENTOS DOS PROJETOS}

Sobre a questão do financiamento, constatamos uma perspectiva animadora, já que muitos dos grupos apresentados tinham financiamento de órgãos de fomento. Isso expressa uma evolução positiva em relação aos grupos participantes dos simpósios anteriores. No III Simpósio, grande parte dos projetos apresentados eram financiados pelo Conselho Nacional de Desenvolvimento Científico e Tecnológico - CNPq, mas também havia alguns financiados pela Coordenação de Aperfeiçoamento de Pessoal de Nível Superior - CAPES, pelas fundações de pesquisas e por agências, ou sob forma de bolsa de pesquisadores do CNPq.

Constatamos, também, estudos consistentes que fazem mapeamentos, tipo estado da arte. Esse tipo de estudo contribui muito para o reconhecimento e compreensão do campo de formação de professores. Sem dúvida, esses trabalhos de estado da arte requerem um número grande de grupos de pesquisa envolvidos, porque não se pode falar em estado da arte quando se faz uma mera revisão de literatura. É impossível, por exemplo, que faça sozinho, um estado da arte, pois esta é uma investigação abrangente, de largo espectro e que exige o envolvimento de vários pesquisadores que dominem a compreensão das tendências de pesquisa no campo. Nesta direção, tivemos a presença de um estudo que fez estado da arte contando com 38 pesquisadores. O que pontuamos é que as políticas de financiamento deveriam considerar a importância desses estudos, bem como dos longitudinais e os de larga escala, que embora requeiram maior despesa, trazem mais valias inestimáveis.

\section{REFERENCIAIS TEÓRICOS E CONCEITOS-CHAVE - AMPLIAÇÃO E DIVERSIFICAÇÃO}

Quanto aos referenciais teóricos, verificamos que estamos trabalhando com um grande número de autores e de conceitos: profissionalidade, identidade profissional, práticas formativas, desenvolvimento profissional, 
conhecimento profissional, aprendizagem da docência, inserção profissional, trabalho docente. Isso remete à constituição do campo teórico, pois, para trabalharmos com esses conceitos, temos recorrido a autores do contexto nacional e a autores do contexto internacional, considerando as questões que estejam sendo analisadas. Por exemplo, a abordagem das narrativas tem sido desenvolvida pelo grupo que está no CIPA, que participa desses congressos, evidenciando a linha italiana, francesa, inglesa. Assim, há uma variedade de referenciais que estão nos orientando nesses trabalhos de pesquisa, favorecendo o aprofundamento dos estudos relativos aos temas investigados. Mas, por exemplo, sobre conhecimento e trabalho docente, o foco tem incidido em Tardif; sobre desenvolvimento profissional, em Imbernón, Nóvoa, Carlos Marcelo Garcia. Desse modo, ocorre uma repetição desses autores e de seus conceitos. Igualmente, temos os nossos colegas de Portugal, muitos dos quais estão presentes aqui nos trabalhos, como Maria do Céu Roldão, que é uma referência frequente nos grupos. Isso não quer dizer que não reconhecemos a contribuição destes autores no campo, contudo a repetição pouco ajuda no aprofundamento de conceitos.

\section{CONSIDERAÇÕES FINAIS}

Ressaltamos a provisoriedade e o limite dessa síntese, que embora tenha sido produzida com a busca do maior rigor possível, ainda carece de maior consistência porque reproduz uma apresentação oral. No entanto, tem um valor histórico, ao registrar um momento específico dos grupos de pesquisa do campo de formação de professores e da pesquisa na área de educação. 